\title{
ON A RELATION BETWEEN INJECTORS AND CERTAIN COMPLEMENTED CHIEF FACTORS OF FINITE SOLUBLE GROUPS
}

Dedicated to the memory of Hanna Neumann

\author{
A. R. MAKAN \\ (Received 27 January 1972) \\ Communicated by G. E. Wall
}

\section{Introduction}

The Fitting class $\mathfrak{S}_{\pi}$ of finite soluble $\pi$-groups, where $\pi$ is an arbitrary set of primes, has the property that each complement of an $\mathfrak{S}_{\pi}$-avoided, complemented chief factor of any finite soluble group $G$ contains an $\Xi_{\pi}$-injector of $G$. In other words, each $\mathfrak{S}_{\pi}$-avoided, complemented chief factor of $G$ is $\mathfrak{S}_{\pi}$-complemented in the sense of Hartley (see [2]).

In general, for a Fitting class $\mathfrak{X}$ of finite soluble groups, none of the complements of an $\mathfrak{X}$-avoided, complemented chief factor of a finite soluble group $G$ may contain an $\mathfrak{X}$-injector of $G$, as an example in Section 2 of [3] shows. As in [3], we will call an $\mathfrak{X}$-avoided, complemented chief factor of $G$ a partially $\mathfrak{X}$-complemented chief factor of $G$ if at least one of its complements contains an $\mathfrak{X}$-injector of $G$. Moreover,

Definimon. A Fitting class $\mathfrak{X}$ of finite soluble groups will be said to have the property $(\Lambda)\left(\left(\Lambda^{*}\right)\right)$ if in each finite soluble group $G$ every $\mathfrak{X}$-avoided, complemented chief factor of $G$ is necessarily a partially $\mathfrak{X}$-complemented (an $\mathfrak{X}$-complemented) chief factor of $G$.

For the rest of the terminology used here we refer the readers to Hartley [2]. All groups considered here are finite and soluble.

Our main purpose of this note is to show that

THEOREM 1.1. A Fischer class has the property $(\Lambda)$ if and only if it is the Fischer class of $\pi$-groups for some suitable set $\pi$ of primes.

In general, one can have a Fitting class which has the property $(\Lambda)$ but which is not $\mathfrak{S}_{n}$ for any set $\pi$ of primes. The normal Fitting class $\mathfrak{H}$ defined in Satz 3.2 of Blessenohl and Gaschütz [1] provides an example of such a Fitting class. 
The $\mathfrak{S}$-injector $V$ of any group $G$ has index at most 2 in $G$. Hence, if $R / S$ is an $\mathfrak{H}$-avoided chief factor of $G$, then $V=V S$ complements $R / S$ in $G$, and so $R / S$ is also a partially $\mathfrak{H}$-complemented chief factor of $G$, Thus, $\mathfrak{H}$ has the property $(\Lambda)$, but it is easy to check that $\mathfrak{S}$ is not $\mathcal{S}_{\pi}$ for any set $\pi$ of primes.

Theorem 1.1 is proved in Section 3 and in Section 2 we discuss Fitting classes with the property $\left(\Lambda^{*}\right)$.

\section{Fitting classes with the property $\left(\Lambda^{*}\right)$.}

In this section, we show that a Fitting class with the property $\left(\Lambda^{*}\right)$ is necessarily $\Xi_{\pi}$ for some suitable set $\pi$ of primes.

THEOREM 2.1. Let $\mathfrak{F}$ be a Fitting class with the property $\left(\Lambda^{*}\right)$. Then $\mathfrak{F}=\mathfrak{S}_{\pi}$ for some suitable set $\pi$ of primes.

Proof. Let $\pi$ be the uniquely determined set of primes such that $\mathfrak{N}_{\pi} \subseteq \mathfrak{F} \subseteq \mathfrak{S}_{x}$ where $\mathfrak{N}_{\pi}$ is the class of all finite nilpotent $\pi$-groups (see Remark 1 of Section 3.3 in Hartley [2]). We show that $\mathfrak{F}=\mathfrak{S}_{\pi}$. Assume to the contrary that $\mathfrak{F} \subset \mathfrak{S}_{\pi}$ and let $G \in S_{\pi} \backslash \mathfrak{F}$ be of minimal order. Since both $\mathcal{S}_{\pi}$ and $\mathfrak{F}$ are Fitting classes, it is clear that $G$ has a unique maximal normal subgroup $M$ of index $p$, say, which belongs to $\mathfrak{F}$. Consider the group $H=G \times G / M$. Clearly $M \times G / M$ is the $\mathfrak{F}$-injector of $H$. Let $G^{*}$ be the subset of $H$ which consists of all elements $(x, x M)$, where $x \in G$. Then $G^{*} \triangleleft H$ and $H=G G^{*}$. In particular, $G^{*} \cap G=M$. Thus, $G^{*}$ complements; $G / M$ in $H$. Since $G / M$ is an $\mathfrak{F}$-avoided, complemented, and hence $\mathfrak{F}$-complemented chief factor of $H$, it follows then that $G^{*}$ contains the $\mathfrak{F}$-injector $M \times G / M$ of $H$. But this is impossible. Hence, we must have $G \in \mathfrak{F}$, and so $\mathfrak{\mho}=\mathfrak{S}_{\pi}$, as required.

In view of Theorem 2.1 and the remark at the beginning of Section 1, we immediately have

Corollary 2.2. A Fitting class has the property $\left(\Lambda^{*}\right)$ if and only if it is the Fitting class of $\pi$-groups for some suitable set $\pi$ of primes.

\section{Proof of the main theorem}

In order to prove Theorem 1.1 we will need the following lemma.

Lemma 3.1. Let $\mathfrak{F}$ be a Fischer class with the property $(\Lambda)$, let $\mathfrak{S}_{p} \subseteq \mathfrak{F}$ and let $G$ be a semidirect product of an $\mathfrak{\mho}$-group $A$ by a cyclic group $B=\langle b\rangle$ of order $p^{n}, n \geqq 1$. Then $G$ is an $\mathfrak{F}$-group.

Proof. Let $C=\langle c\rangle$ be a cyclic group of order $p^{n+1}$, let $H=B \times C$ and let $K$ be the subgroup of $H$ generated by $b c^{p}$. Consider the twisted wreath product (see Neumann [4]) $W$ of $A$ by $H$ over $B \times K$ with the action of $B \times K$ on $A$ being defined as follows: Let $B$ act on $A$ as in the semidirect product $G$ 
of $A$ by $B$, and let $K$ act trivially on $A$. Since $H$ is abelian, it is easy to check that $K$ acts trivially on the base group $D=A_{1} \times A_{c} \times \cdots \times A_{c^{p-1}}$ which is the direct product of $p$ copies of $A$ indexed by the coset representatives $\left\{1, c, \cdots, c^{p-1}\right\}$ of $B \times K$ in $H$, and also $A_{c^{t}}$ is $B$-invariant and $\left[A_{c^{t}}\right] B \cong G$ for $i=0,1, \cdots, p-1$. In particular, $D \times K$ is contained in the $\mathfrak{F}$-injector $V$ of $W$. But then, we must have that $D B / D\left\langle b^{p}\right\rangle$ is an $\mathcal{F}$-covered chief factor of $W$; for, otherwise, it would be an $\mathfrak{F}$-avoided, complemented chief factor of $W$ which is not partially $\mathfrak{F}$-complemented in $W$ since $D K\left\langle b^{p}\right\rangle$ is not contained in any complement of $D B \mid D\left\langle b^{p}\right\rangle$ in $W$. Thus, $V$ covers $D B / D\left\langle b^{p}\right\rangle$. However, since $D\left\langle b^{p}\right\rangle \mid D$ is the Frattini subgroup of $D B / D$, it follows now that $V$, in fact, covers $D B / D$, and so $V \geqq D B$. In particular, $D B \in \mathfrak{r}$ since $D B \triangleleft \triangleleft V$. Finally, since $A_{1}$ is $B$-invariant, and hence also $D B$-invariant, since $A_{1} B / A_{1}$ is a $p$-group and since $\mathfrak{F}$ is a Fischer class, it follows that $G \cong\left[A_{1}\right] B \in \mathfrak{F}$, and the lemma is proved.

We can now complete the proof of Theorem 1.1 as follows:

Proof OF THEOREM 1.1. In view of the remark at the beginning of Section 1, it remains to show that if $\mathfrak{F}$ is a Fischer class with the property $(\Lambda)$, then $\mathfrak{F}$ is the Fischer class of $\pi$-groups for some set $\pi$ of primes. Let $\pi$ be the uniquely determined set of primes such that $\mathfrak{N}_{\pi} \subseteq \mathfrak{F} \subseteq \Im_{\pi}$ (see the proof of Theorem 2.1) We will show that $\mathfrak{F}=\mathfrak{S}_{\pi}$. Assume to the contrary that $\mathfrak{F} \subset \mathfrak{S}_{\pi}$, and let $G \in \mathfrak{S}_{\pi} \backslash \mathfrak{F}$ be of minimal order. Then $G$ has a unique maximal normal subgroup $M$ which lies in $\mathfrak{F}$. Let $|G: M|=p$ and let $x \in G$ be of $p$-power order such that $\langle M, x\rangle=G$. Consider the semidirect product $W$ of $G$ by a cyclic group $\langle\alpha\rangle$ of order $p^{n}=|x|$, the order of $x$ in $G$, with the action of $\langle\alpha\rangle$ on $G$ being given by $g^{\alpha}=g^{x}$ for each $g \in G$. Clearly $M$ is $\langle\alpha\rangle$-invariant, and so, by Lemma 3.1, $[M]\langle\alpha\rangle \in \mathfrak{F}$. Similarly, $[M]\langle\alpha x\rangle \in \mathfrak{F}$. But then

$$
W=[M]\langle\alpha, \alpha x\rangle \in N_{0} \mathfrak{F}=\mathfrak{F},
$$

whence, in particular, $G \in S_{N} \mathscr{F}=\mathscr{F}$, a contradiction. With this contradiction, the proof is complete.

\section{Acknowledgement}

The author is grateful to Dr. R. D. Bercov and Dr. A. H. Rhemtulla for their financial support while this work was done.

\section{References}

[1] D. Blessenohl and W. Gaschütz, 'Über normale Schunck - und Fittingklassen', Math.Z. 118 (1970), 1-8.

[2] B. Hartley, 'On Fischer's dualization of formation theory', Proc. London Math. Soc. (3) 19 (1969), 193-207. 
[3] A. Makan, 'Another characteristic conjugacy class of subgroups of finite soluble groups', $J$. Austral. Math. Soc. 11 (1970), 395-400.

[4] B. H. Neumann, 'Twisted wreath products of groups', Archiv. Math. 14 (1963), 1-6.

The University of Alberta

Edmonton, Alberta

Canada 